Bundesgesundheitsbl 2021 · 64:1125-1135 https://doi.org/10.1007/s00103-021-03392-z Eingegangen: 26. März 2021

Angenommen: 2. Juli 2021

Online publiziert: 16. August 2021

(c) Springer-Verlag GmbH Deutschland, ein Teil von Springer Nature 2021

\section{Zusatzmaterial online}

Zusätzliche Informationen sind in der Online-Version dieses Artikels (https://doi. org/10.1007/s00103-021-03392-z) enthalten.

\section{Hintergrund}

Mit SARS-CoV-2 gelangte ein Coronavirus in Umlauf, für welches die Menschen aufgrund seiner Neuartigkeit nicht über spezifische Abwehrmechanismen des Immunsystems verfügten. COVID-19 manifestierte sich im Dezember 2019 als Häufung schwerer Lungenentzündungen zunächst in Wuhan, China [1]. Am 12.03.2020 erklärte die Weltgesundheitsorganisation (WHO) den COVID19-Ausbruch zur Pandemie [2].

Bei einer weltweiten Gesamtbevölkerung von rund 7,7 Mrd. Menschen im März 2020 [3] wurden seit Pandemiebeginn bis zum 14.02.2021 auf dem WHODashboard insgesamt 108.153.741 bestätigte Fälle und 2.381.295 resultierende Sterbefälle aufgeführt [4]. Dem Robert Koch-Institut (RKI) waren auf der Grundlage der bestehenden gesetzlichen Meldepflichten gemäß $\$ \$ 6$ (ärztliche Meldepflicht) und 7 (Labormeldepflicht) des 2020 angepassten Infektionsschutzgesetzes (IfSG) in diesem Zeitraum bundesweit insgesamt 2.334 .561 bestätigte

Aus Gründen der besseren Lesbarkeit wird in dieser Fachpublikation in der Regel das generische Maskulinum als geschlechtsneutrale Form verwendet.

Sabine Gleich · Susann Schmidt - Doris Wohlrab

Gesundheitsreferat der LH München, München, Deutschland

\title{
COVID-19- und influenzaassoziierte Sterbefälle in München ab März 2020 - eine standardisierte Auswertung von Todesbescheinigungen
}

Fälle und 64.960 Verstorbene gemeldet worden.

Die Pandemie verlief in Deutschland in einer kleineren ersten Welle mit einem zahlenmäßigen Maximum der Neuinfektionen um den 15.03.2020 und einer deutlich größeren zweiten Welle mit einem Maximum an Neuinfektionen um den 20.12.2020 [5]. Laut dem DIVI-Intensivregister der Deutschen Interdisziplinären Vereinigung für Intensiv- und Notfallmedizin waren in genanntem Zeitraum 71.724 COVID-19-Erkrankte intensivmedizinisch behandelt worden.

Schwere Verläufe und Sterbefälle zeigten sich anhand der Meldedaten überwiegend bei hochbetagten Menschen. In der wissenschaftlichen Begründung der Ständigen Impfkommission (STIKO) zur COVID-19-Impfempfehlung wird das hohe Alter als der wesentliche Risikofaktor für einen tödlichen Verlauf dieser Infektionskrankheit genannt [6]. Sehr rasch gelang es der Wissenschaft, Impfstoffe mit unterschiedlichen und teils auch neuartigen Wirkmechanismen $\mathrm{zu}$ entwickeln. Die ersten bedingten Zulassungen für Impfstoffe erfolgten in der Europäischen Union zwischen dem 21.12.2020 und dem 29.01.2021 [7-9].

Die saisonale Influenza verursacht jährlich weltweit zwischen 3 und 5 Mio. Krankheits- und schätzungsweise 290.000-650.000 Todesfälle [10]. Eine 2018 publizierte Metaanalyse kommt zu dem Ergebnis, dass sich jährlich circa $10 \%$ der ungeimpften Erwachsenen mit Influenza infizieren [11]. Die saisonale Influenza mit wechselnden zirkulierenden Varianten erfordert eine jährliche Anpassung der Vakzine. Die Saison 2019/2020 war in Deutschland durch eine parallele dominante Zirkulation von A(H1N1)pdm09und $\mathrm{A}(\mathrm{H} 3 \mathrm{~N} 2)$-Influenzaviren gekennzeichnet, die durch eine geringgradige Zirkulation von Influenza-B-Viren der Viktoria-Linie begleitet wurde [12]. Gemäß aktueller Fassung des IfSG sind nach $\$ 7$ Abs. 1 der direkte Nachweis von Influenzaviren, soweit er auf eine akute Infektion hinweist, sowie nach $₫ 6$ Abs. 1 der Krankheitsverdacht, die Erkrankung sowie der Tod an zoonotischer Influenza namentlich meldepflichtig.

Vor dem Ausrufen der Pandemie durch die WHO [2] entstand die Idee zur Durchführung dieser Studie. Es sollen die Sterbefälle an zwei respiratorisch übertragbaren Infektionskrankheiten (pandemische COVID-19- und saisonale Influenzasterbefälle) mittels der im Gesundheitsamt eingehenden Todesbescheinigungen vergleichend ausgewertet werden.

\section{Methode}

Es erfolgte eine fortlaufende Sichtung aller für den Sterbezeitraum vom 01.03.-31.12.2020 (Kalenderwoche (KW) 10-KW 53) beim Gesundheitsreferat der Landeshauptstadt München (GSR) eingegangenen Todesbescheinigungen (TB). Da alle TB gemäß Art. 3a Bestattungsgesetz (Best $G$ ) bei den für den 


\section{Leitthema}

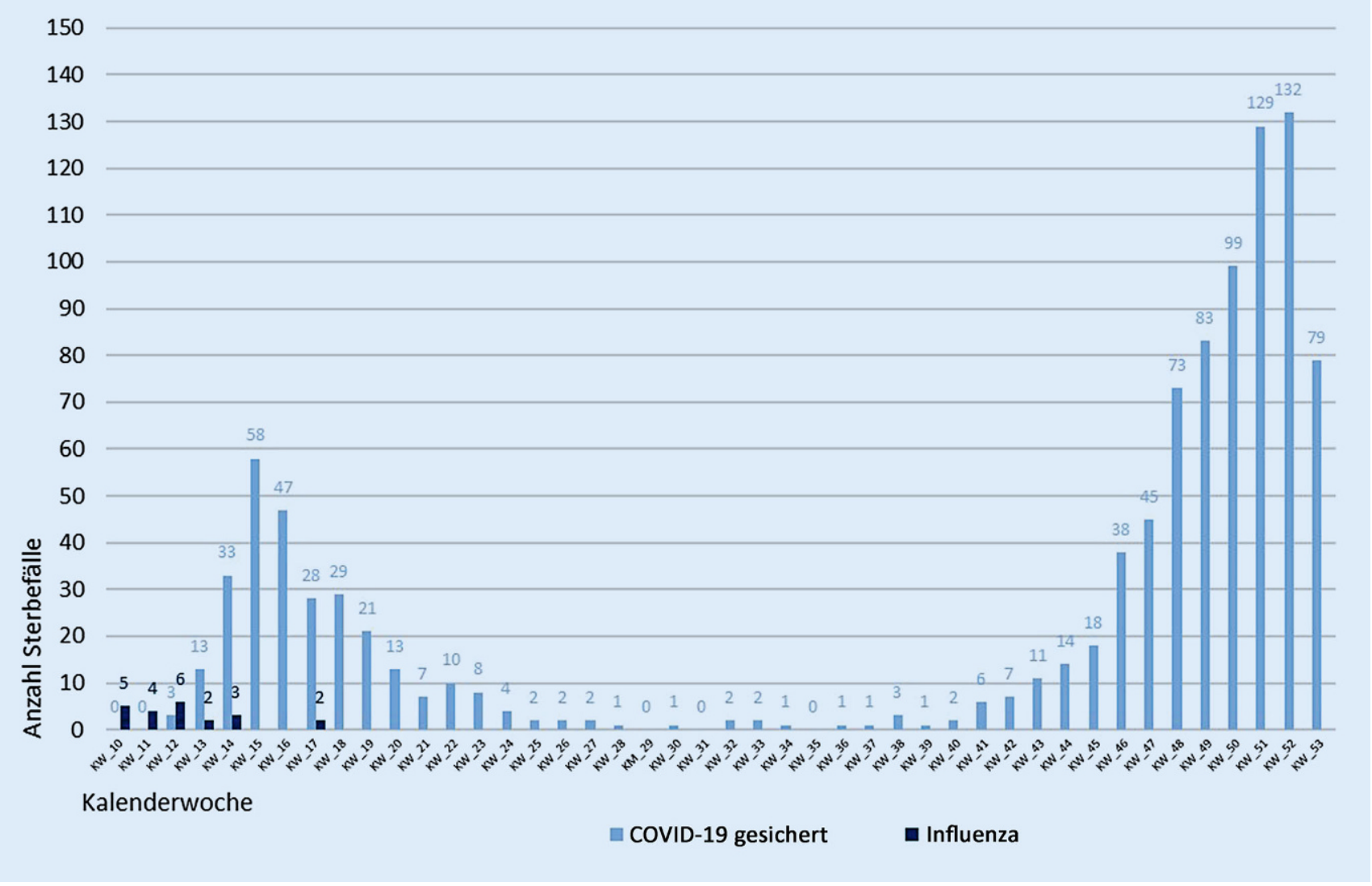

Abb. $1 \Delta$ Zeitlicher Verlauf der COVID-19- und Influenzasterbefälle in München im Untersuchungszeitraum Kalenderwoche (KW) 10 bis KW 53 2020. (Eigene Abbildung)

Sterbeort örtlich zuständigen Gesundheitsämtern archiviert werden, konnte eine vollständige Untersuchung aller Sterbefälle im Stadtgebiet München erfolgen. Diese Fälle umfassen sowohl die Verstorbenen mit Hauptwohnsitz München als auch die mit einem auswärtigen Hauptwohnsitz. Die vorab definierten Einschlusskriterien für die Fallrekrutierung war die Angabe von „COVID-19..., SARS-CoV-2 ...", „Corona ...., ,Influenza (A/B)“ in den Rubriken „Todesursache/Klinischer Befund“ bzw. „weitere bestehende Grunderkrankungen“ der TB.

Bewohner vollstationärer Pflegeeinrichtungen wurden über die in der $\mathrm{TB}$ angegebene Münchner Adresse identifiziert. Eine Einstufung als COVID-19bzw. Influenzafall erfolgte ausschließlich anhand der von den leichenschauenden Ärzten (LS) gemachten Angaben. Alle Daten wurden direkt aus den TB erhoben. Die zu den COVID-19- und Influenzainfektionen erhobenen Daten basieren auf den im Referat auf der Grundlage der $\$ \$ 6,7$ IfSG vorliegenden Meldedaten. Es erfolgten eine standardisierte Eingabe in Microsoft Excel (Microsoft Office 2011, Microsoft Corporation, Redmond, WA, USA) und eine anonymisierte Auswertung auf aggregierter Ebene mit dem Statistikprogramm SPSS (IBM, Armonk, NY, USA) (Version 26.0). Es wurde rein deskriptiv ausgewertet: Kontinuierliche Variablen wurden jeweils mit Mittelwert, Median, Minimum, Maximum und Spannweite angegeben, kategoriale Variablen in Anzahl und Prozent. Um eine grundsätzliche Vergleichbarkeit der beiden Kollektive zu gewährleisten, werden trotz geringer Zahl der Influenzasterbefälle alle erhobenen Daten in absoluten und relativen Zahlen angegeben.

Es wurde kein Ethikkommissionsantraggestellt, da die Auswertung der Daten durch die Gesundheitsämter zur Erfüllung ihrer gesetzlichen Aufgaben nach Art. 3a BestG zulässig ist.

\section{Ergebnisse}

\section{Meldedaten COVID-19- und Influenzainfektionen}

Im Untersuchungszeitraum 01.03.2020 bis 31.12.2020 (KW 10-KW 53) wurden dem GSR insgesamt 44.719 SARS-CoV2- und 2003 Influenzainfektionen gemeldet. An SARS-CoV-2-Infektionen wurden im Zeitraum vom 01.03.-30.04.2020 (KW 10-KW 18) 5739 Fälle, vom 01.05.-30.09.2020 (KW 19-KW 40) 5843 Fälle und vom 01.10.-31.12.2020 (KW 41-53) 33.137 Fälle gemeldet entsprechend der 1. Pandemiewelle, Sommerplateau und 2. Welle. An Influenzainfektionen wurden im Zeitraum von KW 10-KW 181991 Fälle, von KW 19-KW 404 Fälle und von KW 41-53 8 Fälle gemeldet. 
Bundesgesundheitsbl 2021 • 64:1125-1135 https://doi.org/10.1007/s00103-021-03392-z

(c) Springer-Verlag GmbH Deutschland, ein Teil von Springer Nature 2021

\section{S. Gleich · S. Schmidt • D. Wohlrab}

\section{COVID-19- und influenzaassoziierte Sterbefälle in München ab März 2020 - eine standardisierte Auswertung von Todesbescheinigungen}

\section{Zusammenfassung}

Hintergrund. Vor dem Hintergrund der derzeitigen COVID-19-Pandemie entstand die Idee zur Durchführung einer Studie, in der die Sterbefälle an zwei respiratorisch übertragbaren Infektionskrankheiten (pandemische COVID-19 und saisonale Influenza) mittels der im Gesundheitsamt eingehenden Todesbescheinigungen vergleichend ausgewertet werden sollen. Methode. Analysiert wurden die Todesbescheinigungen aller Sterbefälle in München im Sterbezeitraum vom 01.03.-31.12.2020. Die vorab festgelegten Einschlusskriterien waren die Angabe von "Corona, COVID19, SARS-CoV-2, Influenza (A/B)" in den Todesbescheinigungen. Es erfolgte eine standardisierte Dateneingabe, die erhobenen
Daten wurden in aggregierter Form deskriptiv ausgewertet.

Ergebnisse. Im Untersuchungszeitraum verstarben insgesamt 12.441 Personen, 1029 $(8,3 \%)$ an einer gesicherten COVID-19- und $22(0,1 \%)$ an einer Influenzaerkrankung. Die beiden Kollektive stimmten in den untersuchten Parametern gut überein. Das durchschnittliche Sterbealter lag bei rund 80 Jahren, häufigster Sterbeort war die Klinik. Die Infektionskrankheit war in über $90 \%$ der Fälle todesursächlich. Häufigste Todesursachen waren Acute Respiratory Distress Syndrome/respiratorische Insuffizienz und Multiorganversagen. Es wurden durchschnittlich 2 Vorerkrankungen angegeben, am häufigsten Krankheiten des Kreislaufsystems und des Nervensystems. In der zweiten Pandemiewelle war kein Influenzasterbefall zu verzeichnen.

Diskussion. In dieser Untersuchung können erstmals COVID-19- und influenzaassoziierte Sterbefälle vergleichend gegenübergestellt werden. Die Sterbefälle beider Kollektive stimmten in den untersuchten Parametern gut überein, bedürften angesichts der geringen Fallzahlen bei den Influenzafällen aber noch einer Überprüfung in einer größeren Studie.

Schlüsselwörter

SARS-CoV-2 - Pandemie - Öffentlicher Gesundheitsdienst · Todesursachen . Sterbeorte

\section{COVID-19- and influenza-associated deaths in Munich as of March 2020-a standardized analysis of death certificates}

\begin{abstract}
Introduction. In light of the current COVID-19 pandemic, the idea arose to conduct a study to comparatively evaluate deaths from two respiratory transmissible infectious diseases (pandemic COVID-19 and seasonal influenza) by means of death certificates received by the health department.

Methods. Death certificates of all deaths in Munich in the death period from 1 March-31 December 2020 were analyzed. The predefined inclusion criteria were the indication of "Corona, COVID-19, SARS-CoV-2, Influenza (A/B)" on the death certificates. Standardized data entry was performed. The collected data were analyzed descriptively in aggregated form.
\end{abstract}

Results. A total of 12,441 persons died during the study period, 1029 (8.3\%) from confirmed COVID-19 and $22(0.1 \%)$ from influenza. The two collectives matched well in the parameters studied. The mean age at death was approximately 80 years and the most common site of death was in hospital. Infectious disease was the cause of death in more than $90 \%$ of cases. The most common causes of death were acute respiratory distress syndrome/respiratory failure and multiorgan failure. An average of two previous illnesses were reported, most commonly diseases of the circulatory system and nervous system. There was no influenza death in the second pandemic wave.
Discussion. In this study, COVID-19- and influenza-associated deaths were compared for the first time. The deaths of both collectives matched well in the parameters studied, but still require verification in a larger study given the small numbers of influenza cases. An English full-text version of this article is available at SpringerLink as Supplementary Information.

\section{Keywords}

SARS-CoV-2 - Pandemic · Public health service . Causes of death $\cdot$ Places of death

\section{Kollektive Sterbefälle}

Im Untersuchungszeitraum verstarben im Stadtgebiet München insgesamt 12.441 Personen, davon 2014 Personen $(16,2 \%)$ an einer Pneumonie. Bei diesen Sterbefällen waren von den leichenschauenden Ärzten (LS) bei 888 Fällen $(44,1 \%)$ keine Krankheitserreger und bei 34 Fällen (1,7\%) ein bakterieller Erreger angegeben worden. Bei 1029 Fällen $(51,1 \%)$ war von den LS eine gesicherte
COVID-19-Erkrankung, bei 68 Fällen $(3,4 \%)$ der V. a. eine COVID-19-Erkrankung, bei 7 Verstorbenen (0,3\%) eine respiratorische Synzytialvirus(RSV)-Infektion und bei 22 Verstorbenen $(1,1 \%)$ eine Influenzainfektion vermerkt worden. Bei den Influenzatodesfällen wurde vom LS in 14 Fällen eine Influenza A, in 4 Fällen eine Influenza B und in 4 Fällen kein Erreger genannt. Bei keinem Sterbefall war auf der Todesbescheinigung eine SARS-CoV-2-Influenza-Koinfektion vermerkt.

\section{Zeitlicher Verlauf der COVID- 19- und influenzaassoziierten Sterbefälle}

Der zeitliche Verlauf der gesicherten COVID-19- und influenzaassoziierten Sterbefälle kann - Abb. 1 entnommen werden. In der ersten Pandemiewelle kam es in der 15 . KW mit 58 Sterbe- 


\begin{tabular}{|c|c|c|}
\hline Parameter & $\begin{array}{l}\text { Gesicherte COVID-19-Fälle } \\
n=1029(100 \%)\end{array}$ & $\begin{array}{l}\text { Influenzafälle } \\
n=22(100 \%)\end{array}$ \\
\hline \multicolumn{3}{|l|}{ Geschlecht } \\
\hline Männlich & $531(51,6 \%)$ & $11(50,0 \%)$ \\
\hline Weiblich & $498(48,4 \%)$ & $11(50,0 \%)$ \\
\hline \multicolumn{3}{|l|}{ Sterbealter } \\
\hline Mittelwert & 81,7 & 81,6 \\
\hline Median & 83,0 & 84,5 \\
\hline Spannweite & $88(17-105)$ & $51(50-101)$ \\
\hline \multicolumn{3}{|c|}{ Altersgruppen analog LGL } \\
\hline 0-14 Jahre & 0 & 0 \\
\hline 15-34 Jahre & $2(0,2 \%)$ & 0 \\
\hline 35-59 Jahre & $47(4,6 \%)$ & $2(9,1 \%)$ \\
\hline 60-79 Jahre & $311(30,2 \%)$ & $5(22,7 \%)$ \\
\hline$\geq 80$ Jahre & $669(65,0 \%)$ & $15(68,2 \%)$ \\
\hline \multicolumn{3}{|l|}{ Wohnort } \\
\hline $\begin{array}{l}\text { Stadtgebiet Mün- } \\
\text { chen }\end{array}$ & $844(82,0 \%)$ & $18(81,8 \%)$ \\
\hline Außerhalb & $185(18,0 \%)$ & $4(18,2 \%)$ \\
\hline sP-Bewohner ${ }^{\mathrm{a}}$ & $412(40,0 \%)$ & $7(31,8 \%)$ \\
\hline \multicolumn{3}{|l|}{ Sterbeorte } \\
\hline sP-Einrichtung ${ }^{b}$ & $251(24,4 \%)$ & $2(9,1 \%)$ \\
\hline Privatadresse & $39(3,8 \%)$ & $1(4,5 \%)$ \\
\hline Klinik & $739(71,8 \%)$ & $19(86,4 \%)$ \\
\hline Intensivstation & $295(28,7 \%)$ & $6(27,3 \%)$ \\
\hline \multicolumn{3}{|c|}{$\begin{array}{l}\text { a Bewohner einer vollstationären Pflegeeinrichtung } \\
{ }^{\mathrm{b}} \text { Vollstationäre Pflegeeinrichtung } \\
\text { LGL Bayerisches Landesamt für Gesundheit und Lebensmittelsicherheit }\end{array}$} \\
\hline
\end{tabular}

Tab. 2 Ausgewählte Parameter der ausgefüllten Todesbescheinigungen

\begin{tabular}{|c|c|c|}
\hline Parameter & $\begin{array}{l}\text { Gesicherte COVID-19-Fälle } \\
n=1029(100 \%)\end{array}$ & $\begin{array}{l}\text { Influenzafälle } \\
n=22(100 \%)\end{array}$ \\
\hline \multicolumn{3}{|l|}{ Angekreuzter Warnhinweis Infektiosität NVT ${ }^{\mathrm{a}}$} \\
\hline Ja & $951(92,4 \%)$ & $5(22,7 \%)$ \\
\hline \multicolumn{3}{|l|}{ Klare Benennung Krankheit NVT } \\
\hline Ja & $324(31,5 \%)$ & $3(13,6 \%)$ \\
\hline \multicolumn{3}{|l|}{ Todesarten } \\
\hline Natürlich & $961(93,4 \%)$ & $21(95,5 \%)$ \\
\hline Ungeklärt & $52(5,1 \%)$ & $1(4,5 \%)$ \\
\hline Nichtnatürlich & $16(1,6 \%)$ & $0(0,0 \%)$ \\
\hline \multicolumn{3}{|l|}{ Obduktion angestrebt } \\
\hline Ja & $110(10,7 \%)$ & $1(4,5 \%)$ \\
\hline \multicolumn{3}{|l|}{ Obduktion durchgeführt } \\
\hline Ja & $43(4,2 \%)$ & $1(4,5 \%)$ \\
\hline Gerichtlich & $16(1,6 \%)$ & $0(0,0 \%)$ \\
\hline Klinisch-pathologisch & $27(2,6 \%)$ & $1(4,5 \%)$ \\
\hline $\begin{array}{l}\text { Erkrankung todesursächlich (in 1a-c un- } \\
\text { mittelbare Todesursachen) }\end{array}$ & $926(90,0 \%)$ & $21(95,5 \%)$ \\
\hline $\begin{array}{l}\text { Erkrankung nicht todesursächlich (in II } \\
\text { sonstige Erkrankungen) }\end{array}$ & $103(10,0 \%)$ & $1(4,5 \%)$ \\
\hline \multicolumn{3}{|l|}{ a Nichtvertraulicher Teil der Todesbescheinigung } \\
\hline
\end{tabular}

fällen $\mathrm{zu}$ einem ersten, in der zweiten Welle in der 52. KW mit 132 Sterbefällen zu einem zweiten, deutlich ausgeprägteren Maximum. Die in der 53. KW auf den ersten Blick rückläufige Zahl von 73 Sterbefällen hängt damit zusammen, dass methodisch bedingt nur die Fälle der restlichen 4 Wochentage von 2020 berücksichtigt sind. Influenzasterbefälle traten ausschließlich zwischen KW 10 und KW 17 mit der höchsten Zahl an 6 Sterbefällen in KW 12 auf.

\section{Demografische Daten und Sterbeorte}

Der Anteil der Männer an den 1029 gesicherten COVID-19-Sterbefällen lag bei 51,6\% (•Tab. 1). Das durchschnittliche Sterbealter lag bei 81,7 Jahren (Median 83, Minimum 17, Maximum 105). Das Geschlechterverhältnis der 22 Influenzasterbefälle war ausgeglichen. Das durchschnittliche Sterbealter lag hier bei 84,5 Jahren (Median 81,6, Minimum 50, Maximum 101).

Der zeitliche Verlauf der COVID-19Sterbefälle in den verschiedenen Altersgruppen kann $\bullet$ Abb. 2 entnommen werden. In beiden Pandemiewellen verstarben weit überwiegend die ab 80-Jährigen, gefolgt von den 60- bis 79-Jährigen. In München gab es keine Sterbefälle unter 15 Jahren. Der Vergleich mit den Influenzasterbefällen zeigt, dass bei beiden Infektionskrankheiten am häufigsten die Hochbetagten verstarben.

Über $80 \%$ der Verstorbenen beider Kollektive hatten ihren Hauptwohnsitz im Stadtgebiet München, $40 \%$ aller in München verstorbenen COVID-19- und ein Drittel der Influenzasterbefälle waren Bewohner vollstationärer Pflegeeinrichtungen. Häufigster Sterbeort beider Kollektive war die Klinik: bei nahezu allen Influenzasterbefällen und gut $70 \%$ der COVID-19-Sterbefälle. War der Sterbeort ein Krankenhaus, dann trat der Tod in gut einem Viertel der Fälle beider Kollektive auf einer Intensivstation ein. Zweithäufigster Sterbeort der Münchner Sterbefälle waren hiesige Pflegeeinrichtungen - bei einem Viertel der COVID19- und einem Zehntel der Influenzasterbefälle. 


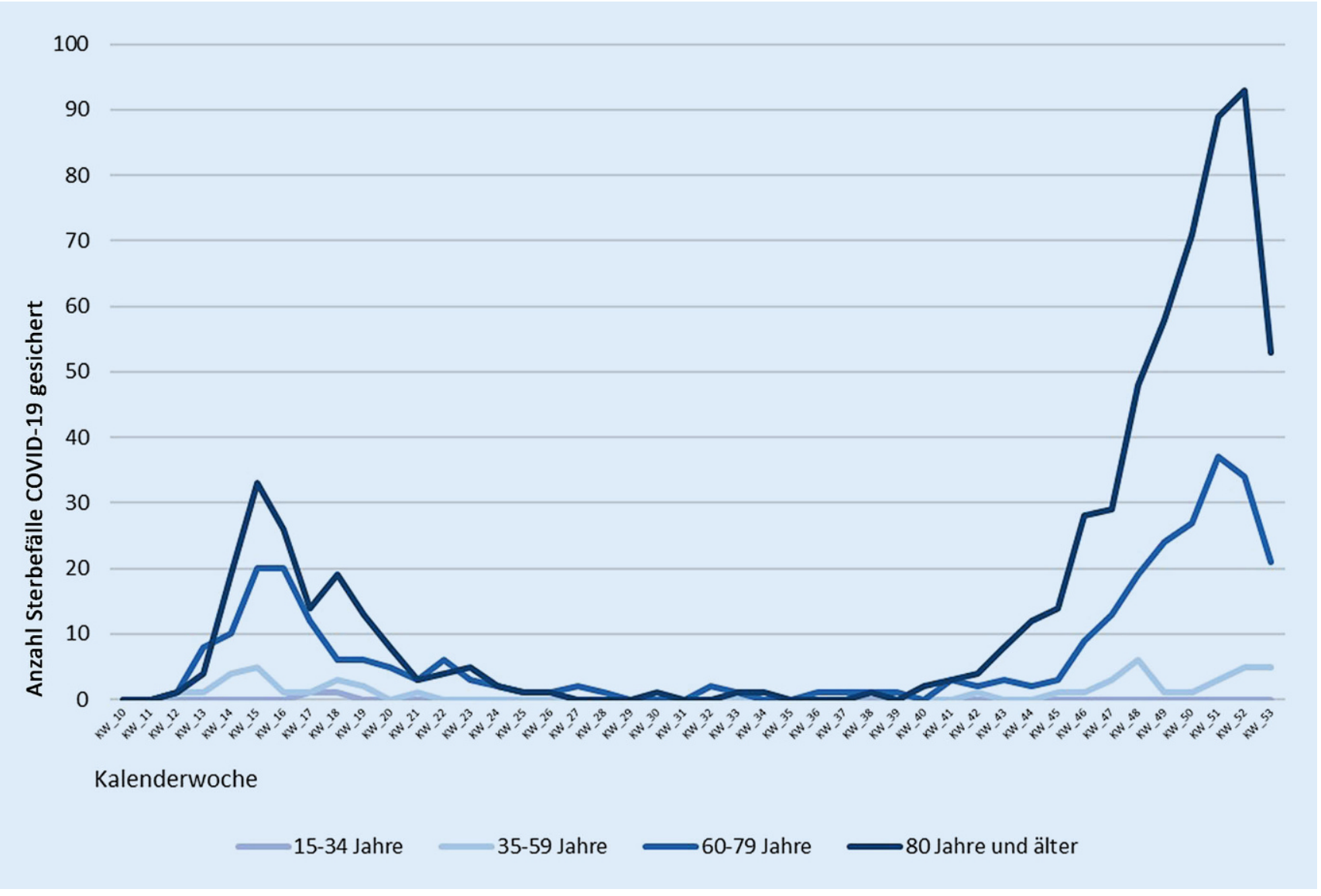

Abb. $2 \Delta$ Altersverteilung der COVID-19-Sterbefälle in München im Untersuchungszeitraum Kalenderwoche (KW) 10 bis KW 53 2020. (Eigene Abbildung)

Ausgewählte Parameter der ausgefüllten Todesbescheinigungen

951 Verstorbene $(92,4 \%)$ der gesicherten COVID-19-Sterbefälle waren vom LS als infektiös gekennzeichnet worden, jedoch nur 5 der Influenzasterbefälle $(22,7 \%$; - Tab. 2). Bei 324 Verstorbenen (31,5\%) des ersten, jedoch bei nur 3 Fällen des zweiten Kollektivs (13,6\%) wurde der Name der Infektionskrankheit im Klartext des nichtvertraulichen Teils der Todesbescheinigung genannt.

In beiden Kollektiven wurde am häufigsten eine natürliche Todesart (961 Fälle, 93,4\% versus 21, 95,5\%), am zweithäufigsten eine ungeklärte Todesart (52 Fälle, 5,1 \% versus 1 Fall, 4,5\%) attestiert. Eine nichtnatürliche Todesart wurde ausschließlich bei den COVID19-Sterbefällen (16 Fälle, 1,6\%) attestiert. Bei diesen Fällen waren von den LS als Begleitumstände innerhalb eines Monats vor dem Versterben durchgeführte ärztliche Eingriffe bzw. Stürze mit Kom- plikationen dokumentiert worden. Die Durchführung einer Obduktion wurde vom LS bei 110 COVID-19-Sterbefällen $(10,7 \%)$ und 1 Influenzasterbefall angestrebt. Tatsächlich obduziert wurden $43(4,2 \%)$ der COVID-19-Sterbefälle, davon $16(1,6 \%)$ gerichtlich nach Anordnung durch die Staatsanwaltschaft München I, jedoch nur 1 Influenzasterbefall.

\section{Todesursachen}

Als todesursächlich - Tod an COVID19 - bewerteten die LS die Erkrankung bei 926 Fällen (90\%), als nicht todesursächlich bei 103 (10\%). Bei den Influenzasterbefällen wurde die Infektion in 21 Fällen $(95,5 \%)$ als todesursächlich gewertet, bei 1 Fall nicht (• Tab. 2).

Am häufigsten verstarben die COVID-19-Erkrankten in 679 Fällen (66,0\%) mit einem Acute Respiratory Distress Syndrome (ARDS) bzw. einer respiratorischen Insuffizienz, in 146 Fäl- len (14,2\%) an Multiorganversagen und in 60 Fällen $(5,8 \%)$ an einer Sepsis (•Tab. 3). Eine ähnliche Häufigkeitsverteilung zeigte sich bei den 22 Influenzasterbefällen: 13 Fälle verstarben an ARDS/respiratorischer Insuffizienz, 7 Fälle an Multiorganversagen und ein Fall an einer Sepsis.

Bei 97 der gesicherten COVID-19Fälle $(9,4 \%)$ und bei einem Influenzasterbefall wurden vom LS Krankheiten des Kreislaufsystems als todesursächlich bewertet, hierbei wurde am häufigsten eine Herzinsuffizienz genannt (50 Fälle, 4,9\% versus ein Fall). Bei 2 SARS-CoV-2-Infizierten $(0,2 \%)$ war das zugrunde liegende Tumorleiden todesursächlich, bei 3 $(0,7 \%)$ ein Suizid. Letztere Fälle befanden sich aufgrund einer psychiatrischen Vorerkrankung in einschlägiger fachärztlicher Behandlung. 


\begin{tabular}{|c|c|c|}
\hline Todesursachen analog ICD-10 ${ }^{a}$ & $\begin{array}{l}\text { Gesicherte COVID-19-Fälle } \\
n=1029(100 \%)\end{array}$ & $\begin{array}{l}\text { Influenzafälle } \\
n=22(100 \%)\end{array}$ \\
\hline Infektionskrankheit (1a-c) & $925(89,9 \%)^{b}$ & $21(95,5 \%)$ \\
\hline ARDS/respiratorische Insuffizienz & $679(66,0 \%)$ & $13(59,1 \%)$ \\
\hline Multiorganversagen & $146(14,2 \%)$ & $7(31,8 \%)$ \\
\hline Sepsis & $60(5,8 \%)$ & $1(4,5 \%)$ \\
\hline Myokarditis & $9(0,9 \%)$ & 0 \\
\hline Herzrhythmusstörungen & $9(0,9 \%)$ & 0 \\
\hline Verdacht bei typischer Symptomatik & $1(0,1 \%)$ & 0 \\
\hline Hirnblutung & $8(0,8 \%)$ & 0 \\
\hline Apoplex & $1(0,1 \%)$ & 0 \\
\hline Lungenembolie & $8(0,8 \%)$ & 0 \\
\hline Hirnödem & $2(0,2 \%)$ & 0 \\
\hline Gastrointestinale Blutung & $2(0,2 \%)$ & 0 \\
\hline Gastroenteritis & $1(0,1 \%)$ & 0 \\
\hline $\begin{array}{l}\text { Krankheiten des Kreislaufsystems } \\
(1 a-c)\end{array}$ & $97(9,4 \%)$ & $1(4,5 \%)$ \\
\hline Herzinsuffizienz & $50(4,9 \%)$ & $1(4,5 \%)$ \\
\hline Myokardinfarkt & $17(1,7 \%)$ & 0 \\
\hline Pulmonale Hypertonie & $2(0,2 \%)$ & 0 \\
\hline Apoplex & $2(0,2 \%)$ & 0 \\
\hline Subarachnoidalblutung & $2(0,2 \%)$ & 0 \\
\hline Intrazerebrale Blutung & $1(0,1 \%)$ & 0 \\
\hline Herzklappenerkrankung & $1(0,1 \%)$ & 0 \\
\hline pAVK & $2(0,2 \%)$ & 0 \\
\hline Lungenembolie & $3(0,3 \%)$ & 0 \\
\hline Sonstige & $17(1,7 \%)$ & 0 \\
\hline Bösartige Neubildungen & $2(0,2 \%)$ & 0 \\
\hline Pankreas & $1(0,1 \%)$ & 0 \\
\hline $\begin{array}{l}\text { Lymphatisches und blutbildendes } \\
\text { System }\end{array}$ & $1(0,1 \%)$ & 0 \\
\hline $\begin{array}{l}\text { Äußere Ursachen von Morbidität } \\
\text { und Mortalität }\end{array}$ & $4(0,4 \%)$ & $0(0,0 \%)$ \\
\hline Suizid & $3(0,3 \%)$ & 0 \\
\hline Unfall & $1(0,1 \%)$ & 0 \\
\hline \multicolumn{3}{|c|}{$\begin{array}{l}\text { ARDS Acute Respiratory Distress Syndrome = akutes Atemnotsyndrom, } p A V K \text { Periphere arterielle Ver- } \\
\text { schlusskrankheit } \\
\text { a } 10 \text {. Revision der Internationalen Statistischen Klassifikation von Krankheiten und verwandter Gesund } \\
\text { heitsprobleme } \\
\text { b Bei einem Fall wurde vom leichenschauenden Arzt bei ungeklärter Todesart keine Todesursache } \\
\text { vermerkt }\end{array}$} \\
\hline
\end{tabular}

\section{Dokumentierte Vorerkrankungen}

In den TB der 1029 gesicherten COVID19-Sterbefälle waren durchschnittlich 1,7 Vorerkrankungen angegeben worden (Median 2, Minimum 0, Maximum 6), in denen der Influenzasterbefälle 1,5 (Median 2, Minimum 0, Maximum 3; - Tab. 4). Bei den COVID-19-Sterbefällen waren am häufigsten Krankheiten des Kreislaufsystems dokumentiert (528 Fälle, $51,3 \%$ ), gefolgt von Krankheiten des

\section{Diskussion}

\section{Fall- und Sterbezahlen, zeitlicher Verlauf}

Dem RKI wurden im Untersuchungszeitraum für Deutschland 1.742.661, für Bayern 328.640 und für München 44.730 Infektionen mit SARS-CoV-2 gemeldet. In diesem Zeitraum verstarben bundesweit 33.624, in Bayern 6765 und 554 Personen mit Hauptwohnsitz München an bzw. mit COVID-19 [5, 13]. In diesem Zeitraum wurden für Deutschland 172.209, für Bayern 48.443 und für München 2003 Influenzainfektionen gemeldet $[14,15]$. Die Pandemie und die damit verbundenen Public-HealthMaßnahmen/Non-pharmaceutical Interventions (NPI) wie AHA-L (Abstandsund Hygieneregeln, (Alltags-)Maske und Lüften) wirkten sich nicht nur auf das Auftreten an SARS-CoV-2-Infektionen, sondern auch auf das Auftreten anderer Infektionskrankheiten aus.

In einer aktuellen Studie des RKI wurde für festgelegte Zeiträume vor und während der COVID-19-Pandemie die relative Veränderung der Fallzahlen von 32 bundesweit meldepflichtigen Infektionskrankheiten berechnet. Diese waren im Vergleich zu den modellbasierten erwarteten Fallzahlen im selben Zeitraum der Vorjahre um insgesamt $35 \%$ rückläufig. Der stärkste Rückgang zeigte sich für respiratorisch übertragbare Krankheiten, für die saisonale Influenza betrug er $-54,4 \%$ [16]. Nach Definition der Arbeitsgemeinschaft Influenza war die Grippewelle der Saison 2019/2020 in der 12. KW 2020 beendet [17].

Analog hierzu war es in München in KW 10-KW 12 zu 22 influenzaassoziierten Sterbefällen gekommen, in der zweiten Pandemiewelle zu keinem einzigen. Durch den Beginn der Studie ab 01.03.2020 wurden mit großer Wahrscheinlichkeit nicht alle Influenzatodesfälle der Grippewelle 2019/2020 in München erfasst, weil die Datenerhebung erst gegen deren Ende begann. Für die COVID-19-Sterbefälle zeigte sich bundesweit ein ähnlicher Verlauf wie in München. 
Tab. 4 In den Todesbescheinigungen dokumentierte Vorerkrankungen

Vorerkrankungen analog ICD-10

Gesicherte COVID-19-Fälle

$n=1029(100 \%)$

\section{Anzah/ Vorerkrankungen}

Mittelwert

Median

Spannweite

A00-B99 Best. infektiöse u. parasitäre Krankheiten

Aspergillose

Chronische Hepatitis C

Tuberkulose und Folgekrankheiten

Pneumokokken

C00-C97 Bösartige Neubildungen

Lymphatisches und blutbildendes System

Genital- und Harnorgane

Bronchien, Lunge

D50-D89 Krankheiten des Blutes, der blutbildenden Organe

Anämie

E00-E90 Endokrine, Ernährungs- u. Stoffwechselkrankheiten

Diabetes mellitus

Adipositas

F00-F99 Psychische und Verhaltensstörungen

Alkoholkrankheit

Nikotinabusus

G00-H95 Krankheiten des Nervensystems und der Sinnesorgane

Alzheimer, Demenz

M. Parkinson

Depression

100-199 Krankheiten des Kreislaufsystems

Arterielle Hypertonie

Koronare Herzerkrankung

Herzinsuffizienz

pAVK ${ }^{b}$

J00-J99 Krankheiten des Atmungssystems

COPD ${ }^{C}$

Asthma bronchiale

Lungenfibrose

K00-K93 Krankheiten des Verdauungssystems

Kachexie

Äthyltoxische Leberzirrhose

1,7

2,0

$6(0-6)$

$26(2,5 \%)$

$6(0,6 \%)$

$4(0,4 \%)$

$2(0,2 \%)$

$2(0,2 \%)$

$142(13,8 \%)$

$36(3,5 \%)$

$31(3,0 \%)$

$19(1,8 \%)$

$18(1,7 \%)$

$13(1,3 \%)$

$176(17,1 \%)$

$154(15,0 \%)$

$9(0,9 \%)$

$4(0,4 \%)$

$25(2,4 \%)$

$13(1,3 \%)$

$10(1,0 \%)$

$342(33,2 \%)$

$275(26,7 \%)$

$32(3,1 \%)$

$12(1,2 \%)$

$528(51,3 \%)$

$209(20,3 \%)$

$209(20,3 \%)$

$133(12,9 \%)$

$51(5,0 \%)$

$92(8,9 \%)$

$78(7,6 \%)$

$6(0,6 \%)$

$6(0,6 \%)$

51 (5,0\%)

$17(1,7 \%)$

$10(1,0 \%)$

$61(5,9 \%)$

webes

Osteoporose

$16(1,6 \%)$

$4(0,4 \%)$

$3(0,3 \%)$

$137(13,3 \%)$

$122(11,9 \%)$

Chronische Niereninsuffizienz
Influenzafälle

$n=22(100 \%)$

1,5

2,0

$3(0-3)$

$1(4,5 \%)$

0

$1(4,5 \%) 0$

0

0

$3(13,6 \%)$

$1(4,5 \%)$

1 (4,5\%)

$1(4,5 \%)$

0

0

$1(4,5 \%)$

$1(4,5 \%)$

0

0

0

0

0

$5(22,7 \%)$

$3(13,6 \%)$

$1(4,5 \%)$

0

$11(50,0 \%)$

$2(9,1 \%)$

$6(27,3 \%)$

$4(18,2 \%)$

$1(4,5 \%)$

$1(4,5 \%)$

$1(4,5 \%)$

0

0

0

0

0

$1(4,5 \%)$

1 (4,5\%)

0

0

$4(18,2 \%)$

$3(13,6 \%)$ 
Risikofaktoren für einen schweren Verlauf, dokumentierte Grunderkrankungen

COVID-19. Als Risikofaktoren für einen schweren Verlauf sind neben hohem Alter [6] beschrieben: männliches $\mathrm{Ge}$ schlecht [18], hohes Alter, Adipositas [19, 20], Rauchen [21] und bestimmte Vorerkrankungen. Dazu zählen Erkrankungen des Herz-Kreislauf-Systems wie arterielle Hypertonie, koronare Herzerkrankung $(\mathrm{KHK})$, zerebrale und peripherere arterielle Verschlusskrankheit, chronische Lungenerkrankungen wie COPD, chronische Nieren- und Lebererkrankungen, Stoffwechselerkrankungen wie Diabetes mellitus, Krebserkrankungen sowie eine Alkoholkrankheit [22-25]. Im Vergleich mit der zitierten Literatur zeigen die hier erhobenen Daten eine gute Übereinstimmung: Das durchschnittliche Sterbealter lag bei 81,7 Jahren, nur knapp $5 \%$ waren jünger als 60 Jahre, über $95 \%$ hingegen älter. Im untersuchten Kollektiv fanden sich alle oben genannten Vorerkrankungen wieder, am häufigsten davon waren Herz-Kreislauf-Erkrankungen bei mehr als der Hälfte der Verstorbenen, gefolgt von Krankheiten des Nervensystems bei einem Drittel und Stoffwechselkrankheiten bei knapp einem Fünftel.

Influenza. Auch bei dieser Infektionskrankheit scheinen Männer ein höheres Risiko für einen schweren Verlauf $\mathrm{zu}$ haben [26], bei den in Deutschland übermittelten Fällen betrug der Anteil der verstorbenen Männer 52\% [27]. Ein höheres Alter erhöht das Risiko ebenfalls: In Deutschland war in der Altersgruppe der über 79-Jährigen nicht nur der Anteil der hospitalisierten Fälle an den übermittelten Fällen, sondern auch der mit einem tödlichen Verlauf mit knapp 5\% am höchsten [27]. An weiteren Faktoren für einen schweren Verlauf werden beschrieben: Übergewicht, Immunsuppression, u.a. nach Transplantationen, Erkrankungen wie Lungenemphysem, chronische Bronchitis, Asthma, Herzinsuffizienz, chronische Leber- und Nierenerkrankungen, rheumatologische Erkrankungen, bösartige Neubildungen und Nikotinabusus [28-31]. Eine Influenza-B-Infektion scheint mit einer höheren Mortalität als eine Influenza-A-Infektion assoziiert zu sein [32]. Im Vergleich mit der zitierten Literatur war bei dem sehr kleinen Kollektiv der Influenzasterbefälle das Geschlechterverhältnis ausgeglichen. Weitere erhobene Daten korrespondierten gut mit verfügbaren Publikationen: Das erhobene durchschnittliche Sterbealter lag bei 84,5 Jahren, nur 2 der 22 Fälle waren jünger als 60 Jahre. Von oben aufgeführten risikoerhöhenden Grunderkrankungen waren am häufigsten Herz-Kreislauf-Erkrankungen, Krankheiten des Nervensystems sowie bösartige Neubildungen vertreten. Eine Influenza-B-Infektion war nur bei 4 Verstorbenen dokumentiert worden. Bei den untersuchten Sterbefällen fand sich kein Fall einer Koinfektion mit SARS-CoV-2 bzw. Influenza. Koinfektionen mit beiden Viren scheinen für die betroffenen Patienten keinen Risikofaktor für einen schweren Verlauf darzustellen [33].

\section{Todesursachen}

COVID-19. Laut Einschätzung der LS war die COVID-19-Erkrankung bei $90 \%$ der Fälle unmittelbar todesursächlich „Tod an COVID-19“. Als die 3 häufigsten Todesursachen wurden ein ARDS/ eine respiratorische Insuffizienz, Multiorganversagen und Sepsis genannt. Diese Zahlen stimmen gut mit einer auf einer Aachener Intensivstation durchgeführten Studie überein. Als todesursächliche Komplikationen traten auch bei den dort behandelten Patienten am häufigsten ARDS/respiratorische Insuffizienz, Multiorganversagen und Sepsis auf [23]. Bei $10 \%$ der Verstorbenen in der vorliegenden Untersuchung war COVID-19 als Begleiterkrankung, also Tod „mit“ COVID-19, genannt worden. Vom LS waren in diesen Fällen meist Erkrankungen des Herz-Kreislauf-Systems genannt, wobei es sich möglicherweise um „Routinediagnosen“ ohne genaue Prüfung gehandelt haben könnte. Eine solche Bewertung ist nach Einschätzung der Autoren jedoch kritisch zu sehen und hätte nur durch eine Obduktion abschließend geklärt werden können. Ein Myokardinfarkt (eine arterielle Thrombose) kann pathophysiologisch auf dem Boden einer vorbestehenden KHK entstehen, er kann sich jedoch auch aufgrund einer COVID19-assoziierten Gerinnungsstörung manifestieren [34, 35]. Bei 2 Fällen waren bösartige Neubildungen, bei 3 Fällen ein Suizid und in einem Fall ein Unfall todesursächlich, nicht jedoch die SARSCoV-2-Infektion.

Influenza. Laut Einschätzung der LS war die Influenzainfektion bei 21 der 22 Fälle unmittelbar todesursächlich. Die in diesem sehr kleinen Kollektiv erhobene Rate liegt damit deutlich höher als die der für die Influenzasaison 2018/2019 bundesweit gemeldeten Sterbefälle. Die Influenzaerkrankung war infolge der von den Gesundheitsämtern in Deutschland übermittelten Daten bei $61 \%$ der infizierten Verstorbenen als unmittelbar todesursächlich bewertet worden [27]. Gründe für diese Abweichung können die sehr kleinen Fallzahlen des untersuchten Kollektivs sein, auch lagen hier nur die TB, jedoch keine Patientenunterlagen für eine differenziertere Beurteilung vor. Alle Verstorbenen waren an einer influenzaassoziierten Pneumonie erkrankt, als häufigste Todesursachen wurde bei 3 Fällen ein ARDS/eine respiratorische Insuffizienz, bei 7 Fällen ein Multiorganversagen und bei 1 Fall eine Sepsis genannt. Bei den Hochbetagten (mehr als 80 Jahre) werden im aktuellen Bericht zur Epidemiologie der Influenza in Deutschland als häufigste Komplikationen eine Pneumonie mit $9,8 \%$, ARDS mit $2,7 \%$ und Beatmung bei $1,1 \%$ genannt [27].

\section{Sterbeorte}

COVID-19. Der Anteil Verstorbener an hospitalisierten COVID-19-Patienten mit schwerer akuter Atemwegserkrankung wurde in einer deutschen SentinelErhebung mit $21 \%$ angegeben, der Anteil beatmeter COVID-19-Intensivpatienten mit $36 \%$ [36]. Eine weitere deutsche Studie stellte fest, dass $22 \%$ der COVID19-Patienten verstarben und die Letalität beatmungspflichtiger Patienten bei $53 \%$ lag [22]. Die Autoren einer internationalen Übersichtsarbeit schätzten den Anteil der Verstorbenen an den intensivmedizinisch behandelten Erkrankten auf $34 \%$ [37]. Nach den Daten dieser Untersuchung verstarben gut $70 \%$ der gesicherten COVID-19-Sterbefälle in 
einer Münchner Klinik, davon nahezu $40 \%$ auf einer Intensivstation. Damit wurde bei an COVID-19 Verstorbenen erwartungsgemäß ein deutlich höherer Anteil des Sterbeortes Klinik als in bisherigen Sterbeortuntersuchungen erhoben: Eine populationsbezogene deutsche Studie aus dem Jahr 2011 stellte fest, dass $51,2 \%$ der Sterbefälle im Krankenhaus verstorben waren [38], ähnliche Zahlen werden aus Kanada und dem Vereinigten Königreich genannt $[39,40]$.

Influenza. Mit 20 von 22 verstarben fast alle Influenzasterbefälle in einer Klinik, davon ein Drittel auf einer Intensivstation. Die Autoren fanden zur Fragestellung, an welchen Sterbeorten Influenzaerkrankte versterben, keine Literaturstellen.

\section{Todesarten}

Eine natürliche Todesart wurde bei rund $94 \%$ der gesicherten COVID-19-Fälle, eine ungeklärte bei $5 \%$ und eine nichtnatürliche bei $1 \%$ bescheinigt. Bei den Influenzasterbefällen zeigte sich bei den attestierten Todesarten eine ähnliche Häufigkeitsverteilung. Eine ungeklärte bzw. nichtnatürliche Todesart war von den LS dann attestiert worden, wenn es in zeitlichem Zusammenhang zum Versterben zu Stürzen bzw. medizinischen Interventionen gekommen war. Diese Zahlen unterscheiden sich von denen einer 2019 publizierten Münchner Studie, damals waren bei allen in München Verstorbenen in $84 \%$ der Fälle eine natürliche und in $16 \%$ eine ungeklärte bzw. nichtnatürliche Todesart bescheinigt worden [41]. Das spricht dafür, dass in nahezu allen Fällen der vorliegenden Untersuchung die Infektionskrankheit vom LS als todesursächlich bewertet wurde und damit die Voraussetzungen für die Attestierung einer natürlichen Todesart gegeben sind.

\section{Obduktionen}

Zur Feststellung der tatsächlichen Todesursache sind Obduktionen unverzichtbar. Erst durch sie und die nachfolgende histopathologische Aufarbeitung entnommener Gewebeproben konnten grundlegende Erkenntnisse für den klinischen Verlauf der COVID-19-Erkran- kung gewonnen werden [34, 35, 42-44]. Das ärztliche Interesse, Obduktionen bei COVID-19-assoziierten Sterbefällen $\mathrm{zu}$ veranlassen, wurde in der Literatur als gering beschrieben [45]. Bei den gesicherten COVID-19-Fällen wurde in München vom LS bei $18 \%$ der Verstorbenen eine Obduktion angestrebt und bei $11 \%$ der Verstorbenen auch durchgeführt. Das erscheint bei einer neuen Infektionskrankheit nicht viel, ist aber immer noch höher als der durchschnittliche bundesweite Wert für Obduktionen von $5 \%$ aller Verstorbenen mit weiter rückläufiger Tendenz [46].

Ein 50-jähriger Influenzasterbefall, bei dem vom LS eine Obduktion angestrebt wurde, wurde klinisch-pathologisch obduziert.

\section{Kennzeichnung als infektiöse Leiche}

An COVID-19 Verstorbene gelten gemäß den Vorgaben des RKI als „kontagiöse“ Leichen [47]. Damit adäquate Schutzmaßnahmen im Umgang mit dem Leichnam ergriffen werden können, ist dieser vom LS beim Ausfüllen der Todesbescheinigung als infektiös zu kennzeichnen. Das erfolgte jedoch nur bei $92 \%$ der Verstorbenen, somit bestand bei den nicht gekennzeichneten Leichen ein potenzielles Infektionsrisiko für Dritte, da dann die Notwendigkeit, eine ausreichende Schutzkleidung zu tragen, nicht zu erkennen ist. Dieses ist jedoch wichtig: Eine Untersuchung aus dem Frankfurter Institut für Rechtsmedizin zeigte, dass die Infektiosität eines an COVID19 Verstorbenen auch nach einem postmortalen Intervall von 17 Tagen nachgewiesen werden kann [48].

Bei den Influenzasterbefällen waren $22 \%$ der Verstorbenen als infektiös gekennzeichnet worden, auch wenn dies nicht den offiziellen Empfehlungen des RKI entspricht [49]. In diesen Fällen mussten also vom Bestatter kostenund zeitaufwendige Schutzmaßnahmen ergriffen werden, die eigentlich nicht erforderlich gewesen wären.

Diese uneinheitliche Vorgehensweise der LS zeigt eine fachliche Unsicherheit, wann ein Verstorbener als infektiös zu kennzeichnen ist. Da der Terminus „in- fektiöse Leiche" jedoch derzeit nicht konkret definiert ist, hatte das GSR zur fachlichen Unterstützung der LS in Abstimmung mit dem Bayerischen Landesamt für Gesundheit und Lebensmittelsicherheit und dem Institut für Rechtsmedizin der Universität München ein Merkblatt mit entsprechenden Hinweisen erarbeitet, welches online abgerufen werden kann [50].

Bei einem Drittel der als infektiös gekennzeichneten COVID-19-Verstorbenen hatte der LS neben dem gesetzten Kreuz „Infektionsgefahr“ auf dem nichtvertraulichen Teil der Todesbescheinigung den Erreger klar benannt - und das, obwohl alle Münchner Ärzte im April 2020 durch die Gesundheitsbehörde zum korrekten Ausfüllen einer Todesbescheinigung informiert und darauf hingewiesen worden waren, dass es aufgrund der über den Tod hinaus bestehenden ärztlichen Schweigepflicht grundsätzlich nicht zulässig ist, im nichtvertraulichen Teil der Todesbescheinigung Krankheitserreger bzw. die zum Tod führenden Diagnosen zu benennen. Offensichtlich besteht zu diesem Punkt noch immer kein Problembewusstsein der LS: Auch 3 der 5 Ärzte, die einen Influenzasterbefall als infektiös deklariert hatten, benannten den Erreger eindeutig. Eine Münchner Untersuchung aus dem Jahr 2019 kam zu dem Ergebnis, dass in $90 \%$ der Kennzeichnungen als „infektiöse Leiche“ der Erreger auf dem nichtvertraulichen Teil der Todesbescheinigung dokumentiert wurde, was als Verstoß gegen die ärztliche Schweigepflicht geahndet werden könnte [51].

\section{Limitationen}

Es handelt sich um eine Untersuchung, bei der alle in München Verstorbenen unabhängig von ihrem Wohnort erfasst wurden. Als Datenquelle standen nur die Angaben der LS in den TB zur Verfügung. Es kann davon ausgegangen werden, dass die Eintragungen teilweise unvollständig sind, sodass sich falsch $\mathrm{zu}$ niedrige Zahlen, beispielsweise bei der Anzahl der angegebenen Begleiterkrankungen, ergeben. Patientenunterlagen standen für die Auswertung nicht zur Verfügung, sodass keine weiteren Infor- 
mationen zum Krankheitsverlauf und zur Diagnostik vorlagen. Somit konnte auch keine Einzelfallprüfung hinsichtlich der Todesursächlichkeit einer COVID19- bzw. Influenzaerkrankung erfolgen. Die Gruppe der Influenzasterbefälle ist im Vergleich zu der der COVID-19Sterbefälle sehr klein, weshalb hier die deskriptiven Auswertungen sehr vorsichtig interpretiert werden müssen. Ebenso wenig ist die tatsächliche Anzahl aller Erkrankten im Untersuchungszeitraum bekannt, somit fehlt der Nenner. Deshalb konnten weitere Parameter wie Prävalenzen und die Letalität nicht berechnet sowie Risikofaktoren nicht statistisch analysiert werden.

\section{Fazit und Ausblick}

In vorliegender Untersuchung wurden erstmals mit COVID-19 und saisonaler Influenza assoziierte Sterbefälle vergleichend gegenübergestellt. Es zeigte sich eine gute Übereinstimmung beider Kollektive in den untersuchten Parametern: So verstarben überwiegend Hochbetagte und viele Bewohner vollstationärer Pflegeeinrichtungen. Die in der Literatur für beide Infektionskrankheiten beschriebenen Risikofaktoren für einen schweren Verlauf wurden auch in dieser Studie gefunden. Im Studienzeitraum gab es übereinstimmend zu den bundesweiten Meldezahlen deutlich weniger Erkrankungen an saisonaler Influenza, in der zweiten COVID-19-Pandemiewelle traten in München keine Influenzasterbefälle auf. Bei den LS bestand, auch bei der neuen Krankheit COVID-19, ein eher geringes Interesse an der Durchführung von Obduktionen. Ebenso zeigten sich fachliche Unsicherheiten der LS bei der Einstufung der Verstorbenen als infektiös, was jedoch erhebliche Auswirkungen auf die Bestattungsmodalitäten hat.

Diese Studie zeigt, dass TB eine wichtige Informationsquelle für den Öffentlichen Gesundheitsdienst darstellen. So können sie einen nicht zu unterschätzenden Beitrag zur Einschätzung des Verlaufes meldepflichtiger Erkrankungen leisten. Hier werden Daten aus dem Großstadtbereich dargestellt, interessant wären auch Untersuchungen aus dem ländlichen Bereich. Ebenso könnte eine systematische Auswertung von TB bislang offene Fragen aus der Versorgungsforschung und Gesundheitsberichterstattung beispielsweise zur Verteilung von Sterbeorten (und damit einem indirekten Zusammenhang mit sozialer Lage) sowie zur Verteilung von Grunderkrankungen beantworten.

\section{Korrespondenzadresse}

PD Dr. med. habil. Sabine Gleich

Gesundheitsreferat der LH München Bayerstr. 28a, 80335 München, Deutschland sabine.gleich@muenchen.de

Danksagung. Die Autorinnen danken Frau Manuela Gleixner und Frau Martina Schubert für die Unterstützung bei der Dateneingabe.

\section{Einhaltung ethischer Richtlinien}

Interessenkonflikt. S. Gleich, S. Schmidt und D. Wohlrab geben an, dass kein Interessenkonflikt besteht.

Für diesen Beitrag wurden von den Autoren keine Studien an Menschen oder Tieren durchgeführt. Für die aufgeführten Studien gelten die jeweils dort angegebenen ethischen Richtlinien.

\section{Literatur}

1. World Health Organization (2020) Coronavirus disease 2019 (COVID-19). Situation report 80. https://apps.who.int/iris/handle/10665/331778? locale-attribute $=$ de \&. Zugegriffen: 29. Okt. 2020

2. World Health Organization (2020) WHO erklärt COVID-19-Ausbruch zur Pandemie. https:// www.euro.who.int/de/health-topics/healthemergencies/coronavirus-covid-19/news/news/ 2020/3/who-announces-covid-19-outbreak-apandemic.Zugegriffen:24. Febr. 2021

3. Statista (2020) Statistiken zur Weltbevölkerung. https://de.statista.com/themen/75/ weltbevoelkerung/.Zugegriffen: 15. Febr. 2021

4. World Health Organization (2021) WHO Coronavirus disease (COVID-19) dashboard. https:// covid19.who.int/.Zugegriffen: 12. Febr. 2021

5. Robert Koch-Institut (2020) Täglicher Lagebericht des RKI zur Coronavirus-Krankheit 2019 (COVID 19). https://www.rki.de/ DE/Content/InfAZ/N/Neuartiges_Coronavirus/ Situationsberichte/Archiv_M\%C3\%A4rz.html.Zugegriffen: 15. Jan. 2021

6. Vygen-Bonnet S, Koch J, Bogdan C, Harder T, Heininger U, Kling K, Littmann M, Meerpohl J, Meyer H, Mertens T, Schmid-Küpke N, Scholz S, Terhardt M, Treskova-Schwarzbach $M$, Überla K, van der Sande M, Wichmann O, Wicker S, Wiedermann U, Wild V, von Kries R (2021) Beschluss der STIKO zur 1. Aktualisierung der COVID-19-Impfempfehlung und die dazugehörige wissenschaftliche Begründung. Epidemiol Bull 2:3-72

7. BioNTech (2020) Press release: Pfizer and BioNTech receive authorization in the European Union for COVID-19 vaccine. https://investors.biontech. $\mathrm{de} /$ news-releases/news-release-details/pfizerand-biontech-receive-authorization-europeanunion-covid.Zugegriffen: 15. Febr. 2021

8. Europäische Kommission (2021) Pressemitteilung, COVID-19: EU-Kommission erteilt Moderna EUweite Zulassung. https://ec.europa.eu/germany/ news/20210106-moderna-zulassung_de.Zugegriffen: 15. Febr. 2021

9. Europäische Kommission (2021) Pressemitteilung, Europäische Kommission erteilt dritte Zulassung für sicheren und wirksamen Impfstoff gegen COVID-19. https://ec.europa.eu/commission/ presscorner/detail/de/ip_21_306. Zugegriffen: 15. Febr. 2021

10. WHO (2018) Influenza (Seasonal). https:// www.who.int/news-room/fact-sheets/detail/ influenza-(seasonal). Zugegriffen: 19. Febr. 2021

11. Somes MP, Turner RM, Dwyer LJ, Newall AT (2018) Estimating the annual attack rate of seasonal influenza among unvaccinated individuals: a systematic review and meta-analysis. Vaccine 36(23):3199-3207

12. Robert Koch-Institut (2020) Virologische Analysen in der Influenzasaison 2019/20. https:// www.rki.de/DE/Content/Infekt/NRZ/Influenza/ zirkulierende/VirolAnalysen_2019_20.html; jsessionid=5ED929FB3A4C4A69043F7ADFE409 2749.internet061\#doc15010308bodyText1. Zugegriffen:25. Mai 2021

13. Landeshauptstadt München (2021) Coronavirusfälle in München: Aktuelle Zahlen. https://www. muenchen.de/rathaus/Stadtinfos/CoronavirusFallzahlen.html.Zugegriffen: 28. Febr. 2021

14. Robert Koch-Institut (2020) Aktuelle Statistik meldepflichtiger Infektionskrankheiten. Epidemiol Bull 12:9

15. Robert Koch-Institut (2021) Aktuelle Statistik meldepflichtiger Infektionskrankheiten. Epidemiol Bull 1:26

16. Buchholz U, Buda S, Prahm K (2020) Abrupter Rückgang der Raten an Atemwegserkrankungen in der deutschen Bevölkerung. Epidemiol Bull $16: 7-9$

17. Robert Koch-Institut (2021) Influenza-Wochenberichte der Arbeitsgemeinschaft Influenza des RKI. https://influenza.rki.de/wochenberichte.aspx.Zugegriffen: 26. Mai 2021

18. Takahashi T, Ellingson MK, Wong $P$, Israelow $B$, Lucas C, Klein J et al (2020) Sex differences in immune responses that underlie COVID-19 disease outcomes. Nature. https://doi.org/10. 1038/s41586-020-2700-3

19. Public Health England (2020) Excess weight and COVID-19: insights from new evidence on the relationship between excess weight and coronavirus (COVID-19). https://www.gov.uk/ government/publications/excess-weight-andcovid-19-insights-from-new-evidence. Zugegriffen: 29. Okt. 2020

20. Palaiodimos L, Kokkinidis DG, Li W, Karamani D, Ognibene J et al (2020) Severe obesity, increasing age and male sex are independently associated with worse in-hospital outcomes, and higher inhospital mortality, in a cohort of patients with COVID-19 in the Bronx, New York. Metabolism 108:154262

21. Vardavas $\mathrm{Cl}$, Nikitara K (2020) COVID-19 and smoking: a systematic review of the evidence. Tob Induc Dis 18:20. https://doi.org/10.18332/tid/ 119324

22. KaragiannidisC,MostertC,HentschkerC, VoshaarT, Malzahn J, Schillinger G et al (2020) Case characteristics, resource use, and outcomes of 
10.021 patients with COVID-19 admitted to 920 German hospitals: an observational study. Lancet RespirMed 8(9):853-862

23. Dreher M, Kersten A, Bickenbach J, Balfanz $P$, Hartmann B et al (2020) Charakteristik von 50 hospitalisierten COVID-19-Patienten mit und ohne ARDS. Dtsch Arztebl Int 117:271-278

24. Wang X, Fang X, Cai Z, Wu X, Gao X et al (2020) Comorbid chronic diseases and acute organ injuries are strongly correlated with disease severity and mortality among COVID-19 patients: a systemic review and meta-analysis. Research (Wash D C). https://doi.org/10.34133/2020/ 2402961

25. Da BL, Im GY, Schiano TD (2020) Covid-19 hangover. A rising tide of alcohol use disorder and alcohol associated liver disease. Hepatology. https://doi. org/10.1002/hep.31307

26. Quandelacy TM, Viboud C, Charu V, Lipsitch M, Goldstein E (2014) Age- and sex-related risk factors for influenza-associated mortality in the United States between 1997-2007. Am J Epidemiol 179(2):156-167

27. Robert Koch-Institut (2019) Bericht zur Epidemiologie der Influenza in Deutschland, Saison 2018/2019. https://edoc.rki.de/handle/176904/ 6253. Zugegriffen: 23 . Febr. 2021

28. Meerhoff TJ, Simaku A, Ulqinaku D, Torosyan L, Gribkova N et al (2015) Surveillance for severe acute respiratory infections (SARI) in hospitals in the WHO European region-an exploratory analysis of risk factors for a severe outcome in influenza-positive SARI cases. BMCInfect Dis 15:1

29. Fezeu L, Julia C, Henegar A, Bitu J, Hu FB, Grobbee DE, Kengne AP, Hercberg S, Czernichow S (2011) Obesity is associated with higher risk of intensive care unit admission and death in influenza $\mathrm{A}(\mathrm{H} 1 \mathrm{~N} 1)$ patients: a systematic review and meta-analysis. Obes Rev 12(8):653-659. https://doi.org/10.1111/j.1467-789X.2011.00864.

30. Liu WD, Yeh CY, Shih MC, Sheng WH (2020) Clinical manifestations and risk factors for mortality of patients with severe influenza during the 2016-2018 season. Int J Infect Dis 95:347-351

31. Murin S, Bilello KS (2005) Respiratory tract infections: another reason not to smoke. Cleve Clin JMed 72(10):916-920

32. Martínez A, Soldevila N, Romero-Tamarit A, Torner N, Godoy P, Rius C, Jané M, Domínguez À (2019) Risk factors associated with severe outcomes in adult hospitalized patients according to influenza type and subtype. PLOS ONE 14(1):e210353

33. Wu X, Cai Y, Huang Xet al (2020) Early release-Coinfection witz SARS-Co-V-2 and influenza $A$ virus in patient with pneumonia, China. Emerg Infect Dis 26(6):1324-1326

34. Dettmeyer R, Lasczkowski G, Weber A, Wolter T, Kernbach-Wighton G (2020) Histopathologische Befunde bei therapierter und nichttherapierter SARS-CoV-2-Infektion - Bericht über 3 Autopsien. Rechtsmedizin 30:336-343

35. Wichmann D, Sperhake JP, Lüthgemann M, Steurer S, Edler Cet al (2020) Autopsy findings and venous thromboembolism in patientes with Covid 19: a prospective cohort study. Ann Intern Med 173(4):268-277. https://doi.org/10.7326/M202003

36. Tolksdorf $\mathrm{K}$, Buda S, Schuler E, Wieler LH, Haas W (2020) Eine höhere Letalität und lange Beatmungsdauer unterscheiden COVID-19 von schwer verlaufenden Atemwegsinfektionen in Grippewellen. Epidemiol Bull 41:3-10
37. Potere N, Valeriani E, Candeloro M, Tana M, Porreca E, Abbate A et al (2020) Acute complications and mortality in hospitalized patients with coronavirus disease 2019: a systematic review and metaanalysis. Crit Care 24(1):389

38. Dasch B, Blum K, Gude P, Bausewein C (2015) Place of death: trends over the course of a decade. A population-based study of death certificates from the years 2001 and 2011. Dtsch Arztebl Int 112(29-30):496-504

39. Motiwala SS, Croxford R, Guerriere DN, Coyte PC (2006) Predictors of place of death for seniors in Ontario: a population-based cohort analysis. Can J Aging 25(4):363-371

40. Ziwary SR, Samad D, Johnson CD, Edwards RT (2017) Impact of place of residence on place of death in Wales: an observational study. BMC Palliat Care 16(1):72. https://doi.org/10.1186/s12904017-0261-5

41. Viehöver S, Peschel O, Graw M, Gleich S (2019) Ordnungswidrigkeiten bei Leichenschau und Ausstellen der Todesbescheinigung Erfahrungen einesGroßstadtgesundheitsamtes. Rechtsmedizin 29:110-116

42. Konopka KE, Wilson A, Myers JL (2020) Postmortem lung findings in a patient with asthma and Coronavirus disease 2019. Chest 158(3):e99-e101. https://doi.org/10.1016/j.chest.2020.04.032

43. Li Y, Xiao SY (2020) Hepatic involvement in COVID19 patients: pathology, pathogenesis and clinical implications. J Med Virol. https://doi.org/10.1002/ jmv. 25973

44. Menter T, Haslbauer JD, Nienhold R, Savic S, Hopfer H et al (2020) Post-mortem examination of COVID-19 patients reveal diffuse alveolar damage with severe capillary congestion and other organs suggesting vascular dysfunction. Histopathology 77(2):198-209. https://doi.org/10.1111/his.14134

45. Pomara C, Volti LG, Cappelo F (2020) COVID19 deaths: Are we sure it is pneumonia? Please, autopsy, autopsy, autopsy! J Clin Med 9(1259):1-3

46. Madea B, WeckbeckerK(2020) Rückläufige Obduktionszahlen in Deutschland. In: Todesfeststellung und Leichenschau für Hausärzte. Springer, Berlin, Heidelberg

47. Robert Koch-Institut (2021) Empfehlungen zum Umgang mit SARS-CoV-2-infizierten Verstorbenen. https://www.rki.de/DE/Content/InfAZ/N/ Neuartiges_Coronavirus/Verstorbene.html.Zugegriffen: 18. Febr. 2021

48. Plenzig S, Bojkova D, Held H, Berger A, Holz F et al (2021) Infectivity of deceased COVID-19 patients. Int J Legal Med. https://doi.org/10.1007/s00414021-02546-7

49. RobertKoch-Institut (2009) Empfehlungen des Robert Koch-Institutes zu Hygienemaßnahmen bei Patienten mit Verdacht auf bzw. nachgewiesener Influenza

50. Landeshauptstadt München, Referat für Gesundheit und Umwelt (2018) Hinweise zum Feld "Infektionsgefahr" auf dem amtlichen Vordruck der Todesbescheinigung. https://www. muenchen.de/rathaus/Stadtverwaltung/Referatfuer-Gesundheit-und-Umwelt/Hygiene_und Umweltmedizin/Sterbewesen.html. Zugegriffen: 18. Febr. 2021

51. Schöpfer J, Stenik A, Eberle L, Koeppel MB, Graw M, Gleich S (2019) Attestierung der Infektionsgefahr bei der Leichenschau Auswertung der Dokumentationsleistung zu meldepflichtigen und nichtmeldepflichtigen infektiösen Krankheiten und Krankheitserregern in Münchner Todesbescheinigungen. Rechtsmedizin 29:190-202 